\title{
EFFECTS OF ONCE-DAILY VERSUS TWICE DAILY DOSING OF CALCIUM FRUCTOBORATE ON KNEE DISCOMFORT. A 90 DAY, DOUBLE-BLIND, PLACEBO CONTROLLED RANDOMIZED CLINICAL STUDY
}

\author{
Z. Pietrzkowski ${ }^{1}$, A. Roldán Mercado-Sesma ${ }^{3}$, R. Argumedo ${ }^{1}$, M. Cervantes $^{1}$, B. Nemzer ${ }^{2}$, T. Reyes-Izquierdo ${ }^{1}$
}

\begin{abstract}
Importance: Previous research showed that a twice-daily $108 \mathrm{mg}$ dose of calcium fructoborate (CFB) improved knee discomfort during a 2-week supplementation period. This current double-blind, placebo-controlled randomized study investigates the effects of CFB supplementation on knee discomfort during 90 days of supplementation. Purpose: To evaluate the comparative effects of once-daily and twice-daily dosing of calcium fructoborate on knee joint discomfort for ninety days. Design: 120 participants with self-reported knee discomfort were recruited and randomized into three groups (each N=40). Participants received: $108 \mathrm{mg}$ CFB twice per day (CFB-G1); or, $216 \mathrm{mg}$ CFB in a single dose (CFB-G2); or, placebo. Setting: Subjects were recruited through advertisement in local papers. The researchers assessed intake and within-study levels of knee discomfort by using the McGill Pain Questionnaire (MPQ) and the Western Ontario and McMaster University Arthritis Index (WOMAC). Results: 62 female and 59 male subjects completed the study. Subjects' average age was (52.84 \pm 8.19 years) and average BMI was (26.76 $\pm 2.50 \mathrm{~kg} / \mathrm{m} 2)$. Statistical differences between groups were calculated using a two-sided, two-sample t-test. Analysis of variance (ANOVA) was used to estimate within-group changes in mean WOMAC and MPQ scores as well as against the control group. When compared to placebo, CFB-G1 showed a significant decrease in reported discomfort on day $14(\mathrm{P}=0.02$, ) day $30(\mathrm{P}=0.003)$, day $60(\mathrm{P}<0.0001)$ and day $90(\mathrm{P}<0.0001)$ according to WOMAC Scores. A similar decrease was observed for CFB-G2 WOMAC Scores on day $14(\mathrm{P}=0.02)$, day $30(\mathrm{P}=0.0003)$, day $60(\mathrm{P}<0.0001)$ and day $90(\mathrm{P}<0.0001)$. When compared to placebo, the MPQ score for CFB-G1 group decreased on day $7(\mathrm{P}=0.002)$, day $14(\mathrm{P}=0.001)$, day $30(\mathrm{P}<0.0001)$, day $60(\mathrm{P}<0.0001)$ and day $90(\mathrm{P}<0.0001)$. MPQ score decreases were also observed for CFB-G2 group on day $7(\mathrm{P}=0.02)$, day $14(\mathrm{P}=0.01)$, day $30(\mathrm{P}<0.0001)$, day $60(\mathrm{P}<0.0001)$ and day $90(\mathrm{P}<0.0001)$. When comparing CFB-G1 and CFB-G2, no significant differences were observed. Importantly, no changes were observed in the WOMAC and MPQ scores within the placebo group. Conclusion: Both CFB groups showed early and significantly improved levels of knee comfort. Knee comfort continued to significantly improve throughout the duration of this 90-day study. No significant differences were observed between the once-daily and the twice-daily doses of CFB.
\end{abstract}

Key words: Calcium fructoborate, knee discomfort, WOMAC, McGill.

\section{Introduction}

Frequent knee pain is a common condition that affects 25 percent of adults. The leading cause of knee pain is a degenerative joint disease, known as degenerative arthritis or osteoarthritis(OA); which is the most prevalent joint disorder in the United States (1). Symptomatic osteoarthritis of the knee affects approximately 10 percent of men and 13 percent of women aged 60 and older (2). The prevalence of

1. Bioresearch Lab, VDF Futureceuticals Inc.; 23 Peters Canyon Rd, Irvine, CA USA 92606; 2. VDF Futureceuticals Inc.; 2692 N. State Rt. 1-17., Momence, IL, USA 60954; 3. Departamento de Salud-Enfermedad, Centro Universitario de Tonalá, Universidad de Guadalajara. Av. Nuevo Periférico No. 555 Ejido San José Tatepozco, C.P. 45425, Tonalá Jalisco, México.

Corresponding Author: Tania Reyes-Izquierdo, 23 Peters Canyon Rd, Irvine, CA, 92606 USA, Phone +1 949502 4496, Fax +1 949502 4987, Email: treyes@ futureceuticals.com

Received August 7, 2017

Accepted for publication November 24, 2017 osteoarthritis of the knee and other sources of knee pain is increasing $(1,3)$. Although OA occurs in people of all ages, it is most commonly found in older people. Common risk factors include increasing age, obesity, previous joint injury, overuse of the joint, weak thigh muscles, and genetics.

According to the Arthritis Foundation, rheumatoid arthritis, gout, psoriatic arthritis, lupus, and fibromyalgia, can also cause knee pain (4). Knee pain not only interferes with an individual's ability to engage in physical exercise, but can also interfere with other basic daily activities. Typically, individuals experiencing knee discomfort have resorted to use of analgesics or non-steroidal anti-inflammatory drugs (NSAIDs) for the relief of symptoms. Unfortunately, NSAIDS have been associated with undesirable side-effects and have been reported to be potentially dangerous for some individuals. Consequently, many active adults prefer a more natural 
solution for their joint discomfort. Therefore, longer-term use of an effective, natural and safe dietary supplement may be a healthier alternative. Previous research suggests that some nutritional supplements such as vitamins (vitamin C and E, D and B), glucosamine, chondroitin sulfates, trace elements (boron, selenium, zinc and copper) and fish oil can improve symptoms of knee discomfort (5-7). More recently, nutraceuticals have been considered as an alternative to stimulate production of needed components of articular cartilage or by slowing down cartilage damage in people with OA (8).

Calcium fructoborate $(\mathrm{CFB})$ is a nature-identical plant mineral complex (a "borocarbohydrate") originally found in certain fruits, vegetables, nuts and legumes, and currently produced by a previously described patented process (9). CFB is a non-animal, generally recognized as safe (GRAS), non-genetically modified organism (GMO), water-soluble material that has been reported to be fast-acting and effective at low doses for relief of joint discomfort (10-14). Our previous research showed that calcium fructoborate significantly improved knee comfort $9,14,15)$ and flexibility through a self-reported Western Ontario and McMaster Universities Index (WOMAC) score $(16,17)$ and McGill Pain Questionnaire (MPQ) index (18-21) during a 2-week supplementation11. This research supported that CFB may provide "fast-acting" relief for joint discomfort if used twice daily at a $108 \mathrm{mg}$ dose (11). However, because discomfort associated with many knee problems generally persists longer than the previouslystudied two-week time-frame, (e.g., in progressive conditions related to osteoarthritis), further investigation was required to measure the longer-term (sub-chronic) effects of CFB on subjects with knee discomfort. In the present study, we examined the effects of once per day (QD) CFB at $216 \mathrm{mg} /$ dose versus twice per day (BID) CFB at $108 \mathrm{mg} /$ dose versus a placebo during ninety days of supplementation. This paper reveals the results of our investigation.

\section{Materials and Methods}

\section{Consent}

This study was conducted according to the Declaration of Helsinki guidelines. All procedures involving human subjects were approved by the Institutional Review Board (Comité de Ética en Investigación Biomédica para el Desarrollo de Fármacos, S.A. de C.V., Av. Sebastian Bach No. 5257, Col. La Estancia, C.P. 45030, Zapopan, JAL, Mexico) (IRB: FCE-NCI-16-06-KNN).

After Institutional Review Board protocol approval, subjects were recruited through advertisement in local papers. Three hundred and sixty male and female subjects were prescreened, according to the inclusion and exclusion criteria. All applicants signed an informed consent form. NutraClinical Inc. (San Diego, CA, USA) performed supplement distribution, sample and data collection according to a protocol designed by BioResearch Lab, VDF FutureCeuticals, Inc. (Irvine, CA, USA).

\section{Materials}

CFB was provided and standardized by VDF FutureCeuticals, Inc., Momence, IL, USA. Silica oxide and fructose were from (Sigma Chem. Co. St. Louis, MO, USA). Capsules were from Capsuline (Pompano Beach, FL, USA), Nalgene ${ }^{\circledR}$ amber bottles were from ThermoFisher Scientific (Waltham, MA, USA).

\section{Inclusion and Exclusion Criteria}

\section{Inclusion criteria}

Subjects who reported knee discomfort for more than 4 weeks prior to enrollment in the study, and who had an initial McGill Score: $>50-<65$ (Average 55.4, $\mathrm{SD} \pm 4.05$, $\mathrm{P}=0.64)$ were included in the study.

Age range: $>35-<65$ years; the average age for the subjects included in the study was 52.8 years of age (SD \pm 8.19).

Other than reported knee discomfort, subjects were generally healthy with no visible evidence of having respiratory or other infections. Subjects were non-diabetic and free of known allergies to dietary products.

No supplements of any kind were permitted within two weeks prior to and during the study period. Participants were advised to abstain from taking vitamin D, testosterone supplements, and steroid-containing prescription or non-prescription medications for 30 days prior to the study period.

Subjects were not included based on the following criteria: Age: $<30$ or $>65$ years, BMI: $<21$ or $>30$; pregnant, nursing, or planning to get pregnant; currently enrolled in another study; subjects with cardiovascular diseases; any knee injury, taking medications for pain or non-steroidal anti-inflammatory drugs (NSAIDs), dietary or nutritional supplements, or vitamin D two weeks prior to the start of this trial.

\section{Study description}

One hundred and fifty-six subjects who satisfied the inclusion criteria were included in the study, with twelve (12) subjects accounted for each group to replace dropouts in order to complete 120 subjects. Subjects were divided into two groups (78 females and 78 males) and by using simple randomization consisting of 78 tokens containing either a number " 1 ", " 2 " or " 3 ". The researchers matched the tokens to a list containing all the participant names and recorded the codes assigned for every supplementation. In order to maintain a doubleblind status, neither the researchers nor the subjects were 
aware of the contents of the capsules. After the study was completed, all the bottles were collected from the subjects (for compliance) and the data was analyzed.

Baseline assessment on Day 1 included a medical history and physical examination for all subjects. Participants underwent blood collections at baseline and on days 7, 14, 30, 60 and 90. Subjects fasted for at least 12 hours prior to blood collection.

Each participant received two bottles containing white capsules and blue/white capsules along with instructions to take the white capsules in the morning and the blue/ white capsules in the afternoon, thirty minutes before meals (breakfast or lunch) and preferably with water. Following a "2-capsule per day" dosage for all groups ensured a comparable perception of all participants being supplemented. Placebo capsules contained 50 $\mathrm{mg}$ of silica/80mg fructose for both, white and blue/ white capsules. CFB-G1 capsules contained 108mg/ capsule of CFB for both white and blue/white capsules. CFB-G2 capsules contained $216 \mathrm{mg}$ of CFB in the white capsules and $50 \mathrm{mg}$ silica/80mg fructose in the blue/ white capsules. On day 1 , all subjects received their test products and were instructed to take first white capsule dose immediately after blood collection. McGill and WOMAC Questionnaires were administered at baseline and at $7,14,30,60$ and 90 days.

\section{Follow up visits}

Each subject received the full number of capsules required for the duration of the study. Subjects received daily telephone calls to assure compliance. As instructed, subjects brought their test bottles to each follow-up visit. During each visit, researchers counted and recorded the remaining number of capsules in the test bottles to ensure compliance.

\section{Rescue medication and concomitant medication}

Subjects were allowed to take acetaminophen only in cases where pain exceeded a value of 6 out of 10 on a provided hospital-type pain scale (simple circle drawings of faces depicting increasing levels of pain). Subjects were provided with Acetaminophen $500 \mathrm{mg} /$ tablet and asked to take a maximum of $1000 \mathrm{mg}$ per day in cases where the pain exceeds tolerability. Participants were instructed to not take this medication within 48 hours of a visit. All the events were recorded in the Rescue Medication Form for every day of the treatment. Subjects were asked to record every Rescue Medication event in the subject's diary. Subjects were asked to take their diaries to each study visit. A summary on subjects taking the Rescue Medication was generated and included in the final study report. Other concomitant medications were recorded in the Concomitant Medication Log Sheet for every day of the treatment.

\section{Blood Collection}

Blood was collected at baseline prior to supplementation and again at days 7, 14, 30, 60 and 90. Samples were always collected under fasting conditions. Two $9 \mathrm{~mL}$ blood samples were drawn from an antecubital vein in anticoagulant-free (dry tubes) (BD Vacutainer Franklin Lakes, NJ, USA) in each participant. Immediately after collection, blood samples were allowed to clot. Serum samples were collected upon clot formation after centrifugation. Serum was aliquoted, snap frozen and kept at $-70^{\circ} \mathrm{C}$ until use.

\section{Blood chemistry}

Blood chemistry was performed on blood samples after every visit. Serum samples collected from each subject at Day 1, Day 60 and Day 90 underwent analysis to monitor any changes during the trial. Analyses included serum glucose, blood urea, nitrogen, creatine, total bilirubin, alkaline phosphatase, total proteins, albumin, globulin, uric acid, calcium, phosphorus, iron, sodium, potassium, chlorine, $\mathrm{CO} 2$, triglyceride, total cholesterol, HDL and LDL. The assays for asparate aminotransferase (AST), alanine aminotransferase (ALT), lactate dehydrogenase (LDH) and gamma-glutamyl transpeptidase (GGTP) were also performed.

\section{McGill Pain Questionnaire}

The McGill Pain Questionnaire (MPQ) is a multidimensional pain questionnaire used to quantify the quality and intensity of pain. This scale contains four subscales consisting of 78 words that participants use to indicate feelings of pain. Participants choose seven words from categories of pain description, pain components, evaluation of pain, and a miscellaneous descriptor category. Each chosen word has an associated numerical value, and total scores range from 0 (no pain) to 78 (severe pain). The McGill Pain Questionnaire was administered at baseline, (and used as part of the inclusion/exclusion criteria), and at 7, 14, 30, 60 and 90 days post-supplementation.

\section{Western Ontario and McMaster Universities Arthritis Index}

The Western Ontario and McMaster Universities Arthritis Index (WOMAC) is a questionnaire used to assess the physical function of joints. WOMAC consists of 24 items divided into 3 subscales, including pain (5 items; scores range from 0 to 20), stiffness (2 items; scores range from 0 to 8 ), and functional limitations (17 items; scores range from 0 to 68). Total scores range from 0 (best) to 96 (worst). The WOMAC index was administered at baseline for all subjects included in the study, and again at 7, 14, 30, 60 and 90 days post-supplementation. 
Table 1

Subjects were randomly assigned to the three study groups. Afterwards, statistical analysis was performed to ensure that there were no significant differences between study groups. Characteristics of study subjects as presented by average values (mean+/-SD) at Day 1 (baseline)

\begin{tabular}{lcccc}
\hline Supplementation & Age (Years) & BMI (kg/m $)$ & WOMAC & MPQ \\
\hline Control & $52.12+/-8.13$ & $25.97+/-2.8 \mathrm{CI}$ & $42.12 \pm 19.28 \mathrm{CI}$ & $54.97 \pm 4.25 \mathrm{CI}$ \\
& $95 \%$ CI $(49.52$ to 54.72$)$ & $95 \%(25.07$ to 26.87$)$ & $95 \%$ (35.95 to 48.29$)$ & $95 \%(53.61$ to 56.33$)$ \\
CFB-G1 & $52.25+/-8.13$ & $26.88+/-2.3 \mathrm{CI}$ & $46.77 \pm 19.90 \mathrm{CI}$ & $55.37 \pm 3.70 \mathrm{CI}$ \\
& $95 \%$ CI 49.64 to 54.85$)$ & $95 \%(26.14$ to 27.61$)$ & $95 \%(40.40$ to 53.14$)$ & $95 \%(54.19$ to 56.56$)$ \\
CFB-G2 & $54.12+/-8.36$ & $27.42+/-2.21$ & $49.58 \pm 18.11$ & $55.82 \pm 4.22$ \\
& $95 \%$ CI $(51.48$ to 56.76$)$ & CI $95 \%(26.72$ to 28.12$)$ & CI $95 \%(43.86$ to 55.30$)$ & CI $95 \%(54.049$ to 57.16 \\
ANOVA & $\mathrm{P}=0.47$ & $\mathrm{P}=0.27$ & $\mathrm{P}=0.21$ & $\mathrm{P}=0.64$ \\
\hline
\end{tabular}

Abbreviations: BMI, body mass index; WOMAC, Western Ontario and McMaster Universities Arthritis index; McGill, McGill Pain Questionnaire; SD, Standard Deviation, $\mathrm{CI}$, confidence intervals.

\section{Data analysis}

\section{Statistical Methods}

All statistical analyses were performed using Graphpad Prism version 6.0. A value $<0.05$ was taken to indicate statistical significance. Statistical differences in the tested groups were calculated by One-Way ANOVA or using a two-sided, two-sample t-test. To address the a priori hypothesis that the supplements would improve mean described discomfort in study subjects with selfreported knee joint pain (and as further confirmed by the intake criteria), the primary analysis tested the effect of treatment on the mean 7-day and 14-day changes from baseline in WOMAC score and McGill score. Subsequent data analysis was also performed for days 30, 60 and 90. A repeated measures analysis of variance (ANOVA) was used to estimate within-group changes in mean WOMAC and MPQ scores over the duration of the study.

\section{Results}

Demographic characteristics of the study population are presented in Table 1. Baseline MPQ values were 55.39 $(\mathrm{SD} \pm 4.05)$ and WOMAC average values were $46.19(\mathrm{SD} \pm$ 19.2). After randomization, One-way analysis of variance (ANOVA) was performed. The age difference between all three groups was not significant $(\mathrm{P}=0.47)$. Since the CFB-G2 group failed the normality test for BMI $(\mathrm{P}=0.03)$. Kolmogolov-Smirnoff normality test was performed for both parameters; age and BMI. There were no significant differences at baseline between groups in either MPQ $(\mathrm{P}=0.64)$ or WOMAC $(\mathrm{P}=0.21)$.

As previously stated, supplements of any kind were not permitted within two weeks prior to and during the study period. Participants were advised to abstain from taking vitamin $\mathrm{D}$, testosterone supplements, and prescription or over-the-counter drugs containing steroids for 30 days prior to the study period. In the placebo group, twenty female and twenty male subjects finished the study, as well as in CF-G1. In CF-G2; twentyone females and twenty males completed the study.

\section{Figure 1}

WOMAC Scores by groups from day 0 [D0] to day 90 [D90]. WOMAC scores were significantly reduced after day 14 and continued to be reduced until D90. No significant differences were observed between CFB-G1 and CFB-G2. Data are presented as score values (mean \pm SEM). $\left[{ }^{*}\right]$ symbol represents statistical significance between Placebo and CFB-G1 and CFB-G2, p<0.05

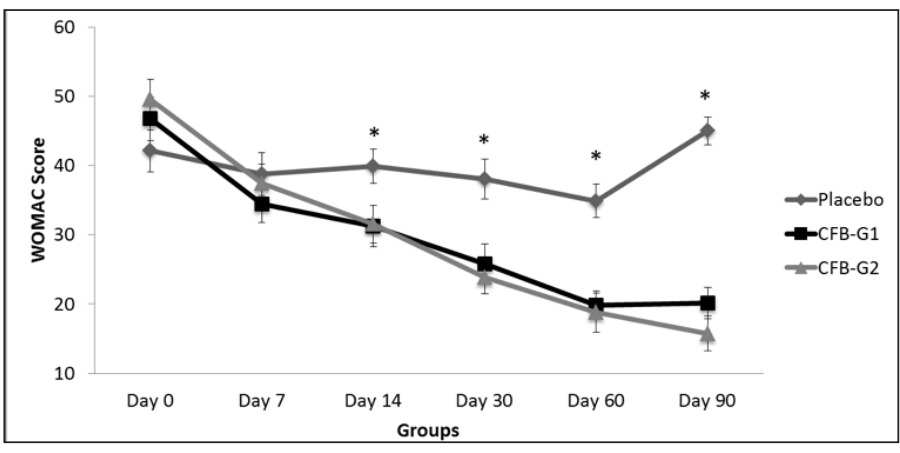

Abbreviations: CFB, calcium fructoborate; WOMAC, Western Ontario and McMAster Universities Arthritis Index; SEM, standard error of the mean.

WOMAC scores are presented in figure 1 . When compared to placebo, CFB-G1 showed a significant decrease on day 14 score $(95 \%$ CI -0.06674 to 17.42$)$ $(\mathrm{P}=0.02)$, day 30 (95\% CI 3.630-20.77) $(\mathrm{P}=0.003)$, day $60(95 \%$ CI 7.254 to 22.85) $(\mathrm{P}<0.0001)$ and day $90(95 \%$ CI 17.62 to 32.13$)(\mathrm{P}<0.0001)$. This decrease was also observed for CFB-G2 on day 14 (95\% CI -0.2749 to 17.10$)$ $(\mathrm{P}=0.02)$, day 30 (95\% CI 5.654 to 22.69$)(\mathrm{P}=0.0003)$, day 60 (95\% CI 8.31 to 23.87) $(\mathrm{P}<0.0001)$ and day $90(95 \%$ CI 22.03 to 36.45) $(\mathrm{P}<0.0001)$. A similar pattern was also observed for MPQ scores. When compared to placebo, the average MPQ score decreased on the CFB-G1 group on day 7 (95\% CI 1.516 to 8.334$)(\mathrm{P}=0.002)$, day $14(95 \% \mathrm{CI} 2.936$ to 11.86$)$ $(\mathrm{P}=0.001)$, day $30(95 \% \mathrm{CI} 7.341$ to 16.31$)(\mathrm{P}<0.0001)$, day $60(95 \%$ CI 10.32 to 19.88$)(\mathrm{P}<0.0001)$ and day $90(95 \%$ 
CI 13.39 to 22.46) $(\mathrm{P}<0.0001)$. This was also observed for the CFB-G2 group on day 7 (95\% CI 0.004905 to 6.780) $(\mathrm{P}=0.02)$, day $14(95 \% \mathrm{CI} 0.5187$ to 9.392$)(\mathrm{P}=0.01)$, day 30 (95\% CI 6.746 to 15.66) $(\mathrm{P}<0.0001)$, day 60 (95\% CI 9.624 to 19.13$)(\mathrm{P}<0.0001)$ and day $90(95 \%$ CI 10.94 to 19.95$)$ $(\mathrm{P}<0.0001)$ (Figure 2). When compared to each other, no significant differences were detected between groups CFB-G1 and CFB-G2.

\section{Figure 2}

MPQ Scores by groups from day 0 [D0] to day 90 [D90]. MPQ scores were significantly reduced at day 7 and continued to be reduced until D90. No significant differences were observed between CFB-G1 and CFBG2. Data are presented as score values (mean $\pm \mathrm{SEM}$ ). [ $\left.{ }^{*}\right]$ symbol represents statistical significance between Placebo and CFB-G1 and CFB-G2, $\mathrm{p}<0.05$

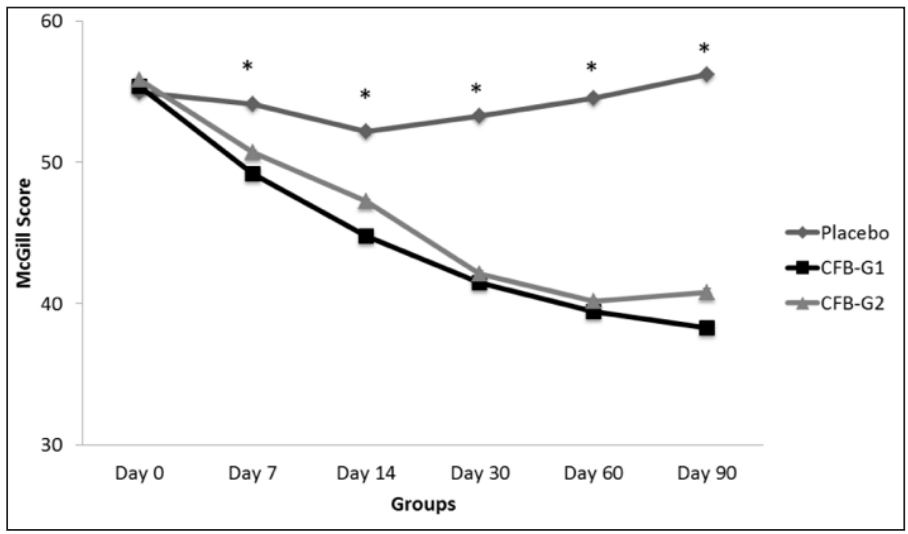

Abbreviations: CFB, calcium fructoborate; MPQ, McGill Pain Questionnsire; SEM, standard error of the mean.

Blood chemistry analysis at day 0, day 60 and day 90 did not indicate any statistically significant changes of key electrolytes, enzymes, lipids and glucose blood levels. All subjects completed this trial without any indications of unusual side effects.

\section{Discussion}

Chronic knee discomfort is a condition that affects the quality of life and impacts mobility. To overcome knee pain, some patients resort to prescription and over-the-counter medications, including opioids or other analgesics to mask pain or steroids to reduce inflammation associated with arthritis (22-24). However, since long-term use of prescription and non-prescription drugs can cause serious side effects $(25,26)$, the use of dietary supplements has been considered as an alternative in the improvement of knee discomfort while reducing the need for NSAIDs.

Previous research supports the use of supplements containing calcium fructoborate (CFB) for fast-acting joint support (10-12). CFB provides knee discomfort relief in as little as 7 days 11 as measured by WOMAC score and McGill index. This study demonstrates the efficacy of CFB for continuous and increasing relief of knee discomfort over a 90-day period. Data herein is in agreement with and extends the results from our previous research on this supplement (10-12). During our previous research, a twice per day CFB dose was provided at $110 \mathrm{mg}$. In this study, we compared the efficacy of a twice-daily (BID) $108 \mathrm{mg}$ dose versus a once-daily (QD) 216mg CFB dose. Our results indicated that both supplementations effectively reduced knee discomfort to a similar extent in WOMAC and McGill scores as compared to placebo. Moreover, no significant differences were observed between both supplemented groups (QD and BID). These results suggest that once-daily dosing may be just as effective as BID and may assure high likelihood of subject compliance. In previous research, calcium fructoborate has not only shown short- and long-term effects on reducing knee discomfort, but also seems to reduce pro-inflammatory and pro-atherogenic markers (27). The effects of CFB on circulating miRNA and on serum biomarkers of inflammation, cartilage and synovium activity are yet to be studied. A future study would help clarify such effects and may help to identify potential mechanisms of action (MOA).

Acknowledgements: We express our gratitude to John Hunter (FutureCeuticals Inc.) for his comments and suggestions in the preparation of this article. We would like to thank Lynn H. for her help in editing the manuscript.

Disclosure Statement: The present study was funded by Futureceuticals, Inc. (Momence IL, USA). All authors declare that they have no conflicts of interest. No competing financial interests exist.

Ethical standards: All procedures performed in this study involving human participants were in accordance with the ethical standards of the institutional review board and with the 1964 Helsinki declaration and its later amendments or comparable ethical standards.

\section{References}

1. Nguyen U-SDT, Zhang Y, Zhu Y, et al. Increasing Prevalence of Knee Pain and Symptomatic Knee Osteoarthritis. Annals of internal medicine. 2011; 155 725-732.

2. Jinks C, Jordan K, Croft P. Measuring the population impact of knee pain and disability with the Western Ontario and McMaster Universities Osteoarthritis Index (WOMAC). Pain. 2002; 100: 55-64.

3. Neugebauer V, Han J, Adwanikar H, Fu Y, Ji G. Techniques for assessing knee joint pain in arthritis. Molecular Pain. 2007; 3: 8.

4. Marks R. Obesity profiles with knee osteoarthritis: correlation with pain disability, disease progression. Obesity (Silver Spring). 2007; 15: 1867-1874.

5. Wang Y, Prentice LF, Vitetta L, Wluka AE, Cicuttini FM. The effect of nutritional supplements on osteoarthritis. Alternative medicine review. 2004 9: 275-296.

6. Gregory PJ, Sperry M, Wilson AF. Dietary supplements for osteoarthritis. Am Fam Physician. 2008; 77.

7. Grover AK, Samson SE. Benefits of antioxidant supplements for knee osteoarthritis: rationale and reality. Nutr J. 2016; 15: 1.

8. French TMC, Daniel O. The utility of neutraceuticals in the treatment of osteoarthritis. Current Rheumatology Reports. 2007; 9: 25-30.

9. Miljkovic D, Scorei RI, Cimpoiasu VM, Scorei ID. Calcium fructoborate: plantbased dietary boron for human nutrition. J Diet Suppl. 2009; 6: 211-226.

10. Reyes-Izquierdo T, Phelan, M.J., Keller, R., Shu, C., Argumedo, R. Pietrzkowski, Z. . Short-term efficacy of a combination of glucosamine and chondroitin sulfate compared toa combination of glucosamine, chondroitin sulfate and calcium fructoborate (CFB) on improvement of knee discomfort conditions in healthy subjects. A comparative, double-blind, placebo controlled acute clinical study. J Aging Res Clin Practice. 2014; 3: 223-228.

11. Pietrzkowski Z, Phelan MJ, Keller R, Shu C, Argumedo R, Reyes-Izquierdo T. Short-term efficacy of calcium fructoborate on subjects with knee discomfort: a comparative, double-blind, placebo-controlled clinical study. Clin Interv Aging. 2014; 9: 895.

12. Reyes-Izquierdo T, Nemzer B, Gonzalez AE, et al. Short-term intake of 
calcium fructoborate improves WOMAC and McGill scores and beneficially modulates biomarkers associated with knee osteoarthritis: a pilot clinical double-blinded placebo-controlled study. Am J Biomed Sci. 2012; 4: 111-122.

13. Scorei ID, Scorei RI. Calcium fructoborate helps control inflammation associated with diminished bone health. Biol Trace Elem Res. 2013; 155: 315321.

14. Scorei R, Mitrut P, Petrisor I, Scorei I. A double-blind, placebo-controlled pilot study to evaluate the effect of calcium fructoborate on systemic inflammation and dyslipidemia markers for middle-aged people with primary osteoarthritis. Biol Trace Elem Res. 2011; 144: 253-263.

15. Scorei RIR, P. Calcium fructoborate--potential anti-inflammatory agent. Biol Trace Elem Res. 2011; 143: 1223-1238.

16. Bellamy N. Pain assessment in osteoarthritis: experience with the WOMAC osteoarthritis index. Semin Arthritis Rheum. 1989; 18: 14-17.

17. Bellamy N, Buchanan WW, Goldsmith CH, Campbell J, Stitt LW. Validation study of WOMAC: a health status instrument for measuring clinically important patient relevant outcomes to antirheumatic drug therapy in patients with osteoarthritis of the hip or knee. J Rheumatol. 1988; 15: 18331840.

18. Melzack R. The McGill Pain Questionnaire: major properties and scoring methods. Pain. 1975; 1: 277-299.

19. Melzack R. Re: Discriminative capacity of the McGill Pain Questionnaire. Pain. 1985; 23: 201-203.
20. Melzack R. The short-form McGill Pain Questionnaire. Pain. 1987; 30: 191-197.

21. Melzack R. The McGill pain questionnaire: from description to measurement. Anesthesiology. 2005; 103: 199-202.

22. James Williams H, Ward JR, Egger MJ, et al. Comparison of naproxen and acetaminophen in a two-year study of treatment of osteoarthritis of the knee. Arthritis \& Rheumatology. 1993; 36: 1196-1206.

23. Zhang W, Jones A, Doherty M. Does paracetamol (acetaminophen) reduce the pain of osteoarthritis?: a meta-analysis of randomised controlled trials. Ann Rheum Dis. 2004; 63: 901-907.

24. Towheed T, Maxwell L, Judd M, Catton M, Hochberg MC, Wells GA. Acetaminophen for osteoarthritis. The Cochrane Library. 2006.

25. Chen Y, Jobanputra P, Barton P, et al. Cyclooxygenase-2 selective nonsteroidal anti-inflammatory drugs (etodolac, meloxicam, celecoxib, rofecoxib, etoricoxib, valdecoxib and lumiracoxib) for osteoarthritis and rheumatoid arthritis: a systematic review and economic evaluation. 2008.

26. Langman MJ, Jensen DM, Watson DJ, et al. Adverse upper gastrointestinal effects of rofecoxib compared with NSAIDs. JAMA. 1999; 282: 1929-1933.

27. Rogoveanu OC, Mogosanu GD, Bejenaru C, et al. Effects of Calcium Fructoborate on Levels of C-Reactive Protein, Total Cholesterol, Low-Density Lipoprotein, Triglycerides, IL-1beta, IL-6, and MCP-1: a Double-blind Placebo-controlled Clinical Study. Biol Trace Elem Res. 2015; 163: 124-131. 